\title{
Expression of mucins MUC5AC and MUC19 on the ocular surface in dry eye syndrome model of ovariectomized female rabbits
}

\author{
Guoxing Li $\mathrm{Li}^{1, A, B}$, Peirong $\mathrm{Lu}^{2, B, C}$, Huiyang Song ${ }^{1, B, C}$, Qinxiang Zheng ${ }^{1, D, E}$, Kaihui Nan ${ }^{1, D-F}$ \\ ${ }^{1}$ Eye Hospital, Wenzhou Medical University, China \\ ${ }^{2}$ Department of Ophthalmology, The First Affiliated Hospital of Soochow University, Suzhou, China \\ A - research concept and design; $\mathrm{B}$ - collection and/or assembly of data; $\mathrm{C}$ - data analysis and interpretation; \\ $D$ - writing the article; $E$ - critical revision of the article; $F$ - final approval of the article
}

\section{Address for correspondence}

Kaihui Nan

E-mail: nankh@eye.ac.cn

\section{Funding sources}

This research was supported with grants from the Natural Science Foundation of Zhejiang Province, China (No. LY17H120003).

Conflict of interest

None declared

Received on February 18, 2017

Revised on March 15, 2017

Accepted on September 26, 2017

Published online on January 14, 2019

Cite as

Li G, Lu P, Song H, Zheng Q, Nan K. Expression of mucins MUC5AC and MUC19 on the ocular surface in dry eye syndrome model of ovariectomized female rabbits. Adv Clin Exp Med. 2019;28(2):165-169. doi:10.17219/acem/78021

\begin{abstract}
Background. Dry eye syndrome (DES) is a common symptom of tear film instability and ocular surface damage due to an abnormal quality and quantity of tears, including the sensation of foreign objects and blurred vision. Among all factors for tear film stability, MUC5AC and MUC19 are very important; the levels of both mucins are associated with the pathogenesis of DES.

Objectives. The aim of this study was to explore the expression of MUC5AC and MUC19 on the ocular surface in a DES model of ovariectomized female rabbits.

Material and methods. Healthy female New Zealand white rabbits ( $n=18$; age: 1 year, weight: $2.5 \pm 0.6 \mathrm{~kg}$ ) were randomly assigned to a test group and a control group. The DES model was constructed in ovariectomized female rabbits. Indicators of ocular surface injury, such as Schirmer's test, corneal fluorescence staining, a conjunctival imprinting cytology test, and the expression of MUC5AC and MUC19 in conjunctival tissues were evaluated with immunohistochemistry in week 1 , week 2 and week 4.

Results. Both the length of soaked test paper and the total scores of corneal fluorescence staining at all time-points were significantly lower in the test group than in the control group, and they decreased over time $(p<0.05)$. The grades of imprinted cells at all time-points were significantly higher in the test group than in the control group, and they increased over time ( $p<0.05$ ). The percentage of goblet cells was significantly lower in the test group than in the control group, and it decreased over time $(p<0.05)$. The percentages of cells with a positive expression of MUC5AC and MUC19 at all time-points were significantly lower in the test group than in the control group, and they decreased over time $(p<0.05)$.
\end{abstract}

Conclusions. The pathogenesis of DES is associated with an increased grade of imprinted cells, decreased goblet cells, and a decreased expression of MUC5AC and MUC19.

Key words: dry eye syndrome model, ovariectomized female rabbits, MUC5AC, MUC19

DOI

10.17219/acem/78021

Copyright

Copyright by Author(s)

This is an article distributed under the terms of the

Creative Commons Attribution Non-Commercial License

(http://creativecommons.org/licenses/by-nc-nd/4.0/) 


\section{Introduction}

Dry eye syndrome (DES) is a common symptom of tear film instability and ocular surface damage due to an abnormal quality and quantity of tears, including the sensation of foreign objects and blurred vision. The prevalence of DES is high in menopausal women, approx. 30-65\%. ${ }^{1}$ The decrease of sex hormones may be the most important reason for the significant increase in DES prevalence in perimenopausal women, which is difficult to treat. ${ }^{2}$ The symptomatic treatment includes artificial tear replacement and extended ocular retention of tears, which can only increase tearing passively. ${ }^{3}$ Recently, autologous submandibular gland transplantation has been somewhat effective, but there may be serious traumatic injury and physiological differences in the liquids secreted by the submandibular and lacrimal glands. ${ }^{4}$ Aragona et al. found that oral pilocarpine hydrochloride could increase the number of goblet cells on the ocular surface and promote the secretion of tearing in patients with Sjögren's syndrome. ${ }^{5}$ Kojima et al. reported that autoserum could significantly improve tear film stability and the scores of fluorescein staining and rose bengal (RB) staining on the ocular surface. ${ }^{6}$ In addition, the immunosuppressant ciclosporin A could also promote the apoptosis of lymphocytes in the lacrimal glands and conjunctivae, and reverse the inflammation on the ocular surface and in the lacrimal glands; however, the local stimulation caused by ciclosporin A limited this application. ${ }^{7}$ Several studies suggested that hormone replacement therapy (HRT) could improve tear function and conjunctival goblet cells ${ }^{8}$; however, since HRT may increase the risk of DES ${ }^{9}$ and may induce the pathogenesis of endometrial carcinoma and mammary cancer as well as androphany and hirsutism, ${ }^{10}$ its use was controversial. It was reported that increased tear osmotic pressure and inflammation were the pathological changes in the progress of DES, and tear film instability was the key factor in the pathological damage of DES and that mucin was one of the necessary active components in tear film. ${ }^{11}$ A change in the quantity and structure of mucin was the intermediate process and one of the results of DES pathogenesis. ${ }^{12}$ Among all factors for tear film stability, MUC5AC and MUC19 were very important: the levels of both mucins were associated with the pathogenesis of DES. ${ }^{13,14}$ Thus, this study aimed to analyze the effect of the expression of MUC5AC and MUC19 on the ocular surface in DES patients, and to provide targets for clinical treatment.

\section{Material and methods}

\section{Material}

This study was in accordance with ethical standards and was approved by the Wenzhou Medical University (China). Healthy female New Zealand white rabbits ( $n=18$; age:
1 year, weight: $2.5 \pm 0.6 \mathrm{~kg}$ ) were purchased from the Shanghai Sangon Experimental Animal Center. These rabbits were fed regularly and were acclimated for 1 week before the experiment. The construction of the DES model of ovariectomized female rabbit consisted of the following: the rabbit was fasted for $12 \mathrm{~h}$; general anesthesia was performed by the administration of ketamine hydrochloride $50 \mathrm{mg} / \mathrm{kg}$ (i.m.); the outer thigh of the hind legs was disinfected with iodophor; the rabbit was placed in the dorsal position and the limbs were fixed; the abdominal cavity was opened through abdominal incision; the bilateral ovarium was resected with an aseptic technique. If the time to tear film rupture (the break-up time (BUT) value) was less than or equal to half the time for normal eyes, the DES model was successfully constructed.

\section{Methods}

The rabbits were randomly assigned to a test group or a control group. Three rabbits in each group at week 1 , week 2 and week 4 were selected; the indicators of ocular surface injury, such as Schirmer's test, corneal fluorescence staining, a conjunctival imprinting cytology test, and the expression of MUC5AC and MUC19 in conjunctival tissues, were evaluated by immunohistochemistry. Schirmer's test consists of placing the Schirmer's test paper in the outer $1 / 3$ boundary of the conjunctival sac in conscious rabbits and suspending the remaining part outside of the conjunctival sac. The paper was retrieved after $5 \mathrm{~min}$ and placed at room temperature for $30 \mathrm{~min}$. The length of soaked test paper was measured with a vernier caliper under microscopy. The procedure for corneal fluorescence staining was as follows: $1 \%$ fluorescein sodium was dropped into the conjunctival sac with a glass rod; after $1 \mathrm{~min}$, the cornea was exposed with a slit lamp and the fluorescent staining of the cornea was detected by cobalt blue light under microscopy; the cornea was divided into 4 quadrants and the intensity of fluorescent staining in each quadrant was graded on a scale from 0 to 3 . The total score was $0-3$ points, i.e., 0 points - no staining; 1 point - disperse and focal staining; 2 points - a cluster of focal staining; 3 points - patchy staining. The procedure for a conjunctival imprinting cytology test was as follows: cellulose filter paper was cut to a size of $5 \times 5 \mathrm{~mm}$ and was immersed in distilled water overnight; it was then placed on the bulbar conjunctiva, pressed for $10 \mathrm{~min}$ and removed; cellulose filter paper was fixed in neutral formaldehyde solution for 10-20 min and stained with the periodic acid-Schiff (PAS) method; the imprinted cells were then observed under light microscopy. Nelson grading criteria (1989) were applied. Squamous metaplasia was graded on a scale of $0-3$ : 0 points - normal; 1 point - mild; 2 points - moderate; 3 points - severe. Goblet cells were counted by randomly selecting 5 fields (left, right, upper, lower, and central) under light microscopy $(\times 200)$ and calculating the percentage of goblet cells. 
The immunohistochemistry testing (Strept Avidin-Biotin Complex (SABC)) was done as follows: anesthesia of the ocular surface was performed with a $0.5 \%$ Benoxil solution; a cellulose acetate membrane $(5 \times 7 \mathrm{~mm})$ was pressed onto the bulbar conjunctiva $2 \mathrm{~mm}$ posterior from the temporal limbus for $50 \mathrm{~s}$; the membrane was then taken and dried; firstly, the membrane was incubated with mouse anti-rabbit MUC5AC and MUC19 mAb (Jiangsu Biyuntian Technology Co., Ltd., Nantong, China), 1:2,000, at $4^{\circ} \mathrm{C}$ overnight and was washed 3 times with phosphate-buffered saline (PBS) for 5 min; the membrane was then incubated with secondary antibody donkey anti-mouse IgG (Jiangsu Biyuntian Technology Co., Ltd.), 1:500, at room temperature for $20 \mathrm{~min}$ and was washed 3 times with PBS for 5 min; next, the membrane was incubated with horseradish peroxidase-labeled streptavidin at room temperature for $20 \mathrm{~min}$ and was washed 3 times with PBS for 5 min; 3,3'-diaminobenzidine (DAB) was added for color development; the membrane was restained with hematoxylin, differentiated with hydrochloric acid alcohol, treated with ammonia water, and washed with distilled water. Five fields were randomly selected under light microscopy $(\times 200)$ to calculate the percentage of positively-stained cells. Phosphate-buffered solution was used instead of the first antibody as a negative control.

\section{Statistical analysis}

SPSS v. 20.0 software (IBM, Inc., Armonk, USA) was used for the statistical analysis. The data is presented as mean and standard deviation (SD). Student's t-test was used for comparison between the 2 independent groups. The one-way analysis of variance (ANOVA) followed by Tukey's post hoc test was used to analyze differences among 3 or more independent groups. Probability value of $\mathrm{p}<0.05$ was considered a significant difference.

\section{Results}

\section{Schirmer's test}

As shown in Table 1, the length of soaked test paper at all time-points was significantly shorter in the test group than in the control group, and it decreased over time $(\mathrm{p}<0.05)$.

\section{Corneal fluorescence staining}

As shown in Table 2, the total scores of corneal fluorescence staining at all time-points were significantly lower in the test group than in the control group, and they decreased over time $(\mathrm{p}<0.05)$.

\section{Conjunctival imprinting cytology test}

As shown in Table 3, the grades of imprinted cells at all time-points were significantly higher in the test group than in the control group, and they increased over time $(\mathrm{p}<0.05)$. The percentage of goblet cells was significantly lower in the test group than in the control group, and it decreased over time $(\mathrm{p}<0.05)$.

Table 1. Schirmer's test results [cm]

\begin{tabular}{|l|c|c|c|c|}
\multicolumn{1}{|c|}{ Group } & Week 1 & Week 2 & Week 4 & F -value \\
\hline Control group & $8.2 \pm 1.3$ & $8.3 \pm 1.4$ & $7.8 \pm 1.5$ & 0.132 \\
\hline Test group & $6.4 \pm 1.2$ & $5.2 \pm 1.3$ & $3.9 \pm 1.4$ & 5.624 \\
\hline $\mathrm{t}$ & 3.965 & 4.527 & 5.327 & - \\
\hline $\mathrm{p}$ & 0.035 & 0.029 & - & - \\
\hline $\mathrm{t}, \mathrm{p}$ - Student's t-test; F, p-value - one-way analysis of variance (ANOVA), followed by Tukey's post hoc test.
\end{tabular}

Table 2. Corneal fluorescence staining

\begin{tabular}{|l|c|c|c|c|}
\hline \multicolumn{1}{|c|}{ Group } & Week 1 & Week 2 & Week 4 & F-value \\
\hline Control group & $10.5 \pm 2.3$ & $10.3 \pm 2.4$ & $10.4 \pm 2.6$ & 0.096 \\
\hline Test group & $7.8 \pm 1.5$ & $5.9 \pm 1.3$ & $4.1 \pm 1.4$ & 5.432 \\
\hline $\mathrm{t}$ & 3.857 & 4.628 & 5.714 & - \\
\hline $\mathrm{p}$ & 0.036 & 0.026 & 0.017 & - \\
\hline
\end{tabular}

Table 3. Conjunctival imprinting cytology test

\begin{tabular}{|c|c|c|c|c|c|c|c|c|c|c|}
\hline \multirow{2}{*}{ Group } & \multicolumn{5}{|c|}{ Grade of imprinted cells } & \multicolumn{5}{|c|}{ Percentage of goblet cells } \\
\hline & week 1 & week 2 & week 4 & $\mathrm{~F}$ & $p$-value & week 1 & week 2 & week 4 & $\mathrm{~F}$ & $p$-value \\
\hline Control group & $0.8 \pm 0.2$ & $1.2 \pm 0.3$ & $1.0 \pm 0.4$ & 0.241 & 0.758 & $42.5 \pm 6.5$ & $43.6 \pm 7.2$ & $44.8 \pm 7.9$ & 0.326 & 0.721 \\
\hline Test group & $1.5 \pm 0.3$ & $1.8 \pm 0.4$ & $2.3 \pm 0.5$ & 5.327 & 0.028 & $35.4 \pm 10.2$ & $31.2 \pm 11.3$ & $25.6 \pm 12.2$ & 5.764 & 0.013 \\
\hline t & 3.625 & 4.721 & 5.285 & - & - & 4.325 & 4.869 & 5.326 & - & - \\
\hline$p$ & 0.039 & 0.025 & 0.017 & - & - & 0.032 & 0.027 & 0.021 & - & - \\
\hline
\end{tabular}


Table 4. The percentages of cells with positive expression of MUC5AC and MUC19

\begin{tabular}{|c|c|c|c|c|c|c|c|c|c|c|}
\hline \multirow{2}{*}{ Group } & \multicolumn{5}{|c|}{ MUC5AC } & \multicolumn{5}{|c|}{ MUC19 } \\
\hline & week 1 & week 2 & week 4 & $\mathrm{~F}$ & $p$-value & week 1 & week 2 & week 4 & $\mathrm{~F}$ & p-value \\
\hline Control group & $35.9 \pm 12.3$ & $36.7 \pm 13.4$ & $34.8 \pm 15.2$ & 0.165 & 0.825 & $41.2 \pm 13.6$ & $38.9 \pm 14.2$ & $37.5 \pm 15.7$ & 0.215 & 0.832 \\
\hline Test group & $29.5 \pm 12.6$ & $26.3 \pm 11.4$ & $21.2 \pm 12.3$ & 5.217 & 0.026 & $30.5 \pm 12.5$ & $27.6 \pm 11.7$ & $23.4 \pm 12.3$ & 5.525 & 0.016 \\
\hline $\mathrm{t}$ & 3.654 & 3.987 & 4.432 & - & - & 4.127 & 4.659 & 4.897 & - & - \\
\hline$p$ & 0.038 & 0.035 & 0.028 & - & - & 0.036 & 0.033 & 0.028 & - & - \\
\hline
\end{tabular}

\section{The expression of MUC5AC and MUC19}

As shown in Table 4, the percentages of cells with a positive expression of MUC5AC and MUC19 at all time-points were significantly lower in the test group than in the control group, and they decreased over time $(\mathrm{p}<0.05)$.

\section{Discussion}

Proteins are the final effectors of genes and they directly reflect the complexity and diversity of biological activities. Mucin is produced in the conjunctiva and apparatus lacrimalis, and is connected to the oligosaccharide side chain through hydrophilic oxygen. Glycosylation provides an electrical charge to mucin, which forms a hydrogen bond with $\mathrm{H}_{2} \mathrm{O}$; thus, highly hydrophilic gel is formed to prevent dryness on the ocular surface. The level of mucin on the ocular surface can be used to evaluate the function of the tear film. MUC5AC is the mucin formed by high-molecular-weight gel and secreted by conjunctival goblet cells. Zhao et al. reported that the level of MUC5AC was significantly lower in patients with DES than in the normal population. ${ }^{15}$ Corrales et al. reported that the level of MUC5AC was significantly lower in patients with DES and was associated with the degree of corneal fluorescence staining. ${ }^{16}$ Zhang et al. found that the expression of MUC5AC was positively associated with the time to tear film rupture. ${ }^{17}$ MUC19 was highly expressed in normal tear gland tissues, conjunctival goblet cells and corneal and conjunctival epithelial cells, yet the expression of MUC19 was significantly decreased in patients with DES; the decrease was similar to that of MUC5AC. ${ }^{18}$

Watanabe reported that changes in the expression levels of both the transmembrane mucin MUC5AC produced in corneal and conjunctival epithelial cells and the secretory gel-like mucin MUC19 produced in goblet cells could directly impact the stability of tear film. ${ }^{19}$ Moreover, the stability of tear film was also dependent on the normal functioning of the neural reflex system, which controls the secretion of the tear gland and the eyelid movement of blinking, namely the first branch of the trigeminal nerve and the facial nerve. The neurons containing vasoactive intestinal peptides (VIPs) were distributed around conjunctival goblet cells; both in vitro and in vivo studies confirmed that VIPs could promote the secretion of mucin in conjunctival goblet cells. In contrast, when the number and function of conjunctival goblet cells were abnormal, the secretion of mucin in tears was abnormal as well, and tear film was less stable. As a result, the production of tears on the ocular surface became abnormal, leading to eye discomfort and decreased vision.

This study showed that both the length of soaked test paper and the total scores of corneal fluorescence staining at all time-points were significantly lower in the test group than in the control group, and that they decreased over time $(\mathrm{p}<0.05)$; the grades of imprinted cells at all time-points were significantly higher in the test group than in the control group, and they increased over time $(\mathrm{p}<0.05)$; the percentage of goblet cells was significantly lower in the test group than in the control group, and it decreased over time $(p<0.05)$; the percentages of cells with a positive expression of MUC5AC and MUC19 at all time-points were significantly lower in the test group than in the control group, and they decreased over time $(p<0.05)$. These results indicated that the pathogenesis of DES was associated with a greater number of imprinted cells, fewer goblet cells and a decreased expression of MUC5AC and MUC19.

Etiological treatment may be the best treatment of diseases. It has been reported that both the immediate and persistent effects of acupuncture were better than that of artificial tears in the treatment of DES. ${ }^{20}$ Acupuncture was characterized by little to no trauma, and it could promote the active secretion of tears from the tear glands, which might increase the level of VIPs in the tear glands and the expression of MUC5AC and MUC19.

\section{References}

1. Wan $\mathrm{KH}, \mathrm{Chen} \mathrm{LJ}$, Young AL. Depression and anxiety in dry eye disease: A systematic review and meta-analysis. Eye (Lond). 2016;30(12): 1558-1567.

2. Sriprasert I, Warren DW, Mircheff AK, et al. Dry eye in postmenopausal women: A hormonal disorder. Menopause. 2016;23(3):343-351.

3. Ching-Li T, Ya-Jung $\mathrm{H}, \mathrm{Chen} \mathrm{ZY}$, et al. Synergistic effect of artificial tears containing epigallocatechin gallate and hyaluronic acid for the treatment of rabbits with dry eye syndrome. PLoS One. 2016;11(6): e0157982.

4. Yu GY, Zhu ZH, Mao C, et al. Microvascular autologous submandibular gland transfer in severe cases of keratoconjunctivitis sicca. Maxillofac Plast Reconstruct Surg. 2015;33:235-239.

5. Aragona P, Pietro RD, Spinella R, et al. Conjunctival epithelium improvement after systemic pilocarpine in patients with Sjogren's syndrome. Brit J Ophthalmol. 2006;90:166-170.

6. Kojima $T$, Ishida R, Dogru M, et al. The effect of autologous serum eyedrops in the treatment of severe dry eye disease: A prospective randomized case-control study. Am J Ophthalmol. 2005;139:242-246. 
7. Yavuz B, Pehlivan SB, Kaffashi A, et al. In vivo tissue distribution and efficacy studies for cyclosporin A loaded nano-decorated subconjunctival implants. Drug Deliv. 2016;23(9):3279-3284.

8. Feng Y, Feng G, Peng S, et al. The effect of hormone replacement therapy on dry eye syndrome evaluated with Schirmer test and break-up time. J Ophthalmol. 2015;2015:420302.

9. Feng Y, Feng G, Peng S, et al. The effects of hormone replacement therapy on dry eye syndromes evaluated by Schirmer test depend on patient age. Cont Lens Anterior Eye. 2015;39:124-127.

10. Erdem U, Ozdegirmenci $O$, Sobaci E, et al. Dry eye in post-menopausal women using hormone replacement therapy. Maturitas. 2007;56: 257-262.

11. Ablamowicz AF, Nichols JJ. Ocular surface membrane-associated mucins. Ocul Surf. 2016;14:331-341.

12. Uchino $\mathrm{Y}$, Uchino $\mathrm{M}$, Yokoi $\mathrm{N}$, et al. Impact of cigarette smoking on tear function and correlation between conjunctival goblet cells and tear MUC5AC concentration in office workers. Sci Rep. 2016;6. Article number: 27699.

13. Coursey TG, Henriksson JT, Barbosa FL, et al. Interferon- $\gamma$-induced unfolded protein response in conjunctival goblet cells as a cause of mucin deficiency in Sjögren syndrome. Am J Pathol. 2016;186: 1547-1558.
14. Lin H, Qu Y, Geng Z, et al. Air exposure induced characteristics of dry eye in conjunctival tissue culture. PLoS One. 2014;9:e87368.

15. Zhao H, Jumblatt JE, Wood TO, et al. Quantification of MUC5AC protein in human tears. Cornea. 2001;20:873-877.

16. Corrales RM, Narayanan S, Fernández I, et al. Ocular mucin gene expression levels as biomarkers for the diagnosis of dry eye syndrome. Invest Ophthalmol Vis Sci. 2011;52:8363-8369.

17. Zhang J, Yan X, Li H. Analysis of the correlations of mucins, inflammatory markers, and clinical tests in dry eye. Cornea. 2013;32:928-932.

18. Yu DF, Chen Y, Han JM, et al. MUC19 expression in human ocular surface and lacrimal gland and its alteration in Sjögren syndrome patients. Exp Eye Res. 2008;86:403-411.

19. Watanabe H. Significance of mucin on the ocular surface. Cornea. 2002;21:17-22.

20. Yang $L$, Yang $Z$, Yu $H$, et al. Acupuncture therapy is more effective than artificial tears for dry eye syndrome: Evidence based on a meta-analysis. Evid Based Complement Alternat Med. 2015;2015:143858. 